\title{
Le récepteur du PYY: un élément important du système antilipolytique de l'adipocyte
}

Le peptide YY (PYY) est une hormone intestinale présentant $70 \%$ d'analogie avec le neuropeptide Y (NPY). Le PYY est libéré lors des repas, alors que le NPY est localisé dans les systèmes nerveux central et périphérique. Leurs récepteurs appartiennent à la famille des protéines à sept passages transmembranaires. Dans l'adipocyte, ces récepteurs sont couplés aux protéines $G$, ici aux protéines $\mathrm{Gi}$ inhibitrices de l'activité de l'adénylyl cyclase. Par cet intermédiaire, les PYY et NPY se comportent comme de puissants inhibiteurs de la lipolyse, cet effet variant cependant en fonction des espèces et de la localisation des dépôts adipeux.

Le tissu adipeux assure le maintien de l'équilibre énergétique de l'organisme par l'intermédiaire de deux voies métaboliques essentielles: la lipogenèse (synthèse des triglycérides) et la lipolyse (hydrolyse des triglycérides en glycérol et acides gras libres). Ces fonctions sont sous le contrôle de diverses hormones et neurotransmetteurs. Ainsi, l'insuline est l'hormone lipogénique et antilipolytique alors que l'ACTH et le glucagon peuvent exercer des effets lipolytiques chez certaines espèces. Les catécholamines (adrénaline, noradrénaline) jouent aussi un rôle important et complexe dans la régulation de la lipolyse; selon les conditions elles peuvent exercer des effets lipolytiques ou antilipolytiques. En effet, l'adipocyte a la particularité d'exprimer à la fois des récepteurs $\beta$-adrénergiques (lipolytiques) des protéines Gs ainsi que des récepteurs $\alpha_{2}$-adrénergiques (antilipolytiques), inhibiteurs de cette enzyme via des protéines Gi (figure 1). D'autres agents endogènes inhibiteurs de l'activité de l'adénylyl cyclase ont également été décrits ; il s'agit de l'adénosine et des prostaglandines. Leurs effets, très puissants, ont été mis en évidence sur des adipocytes isolés de différentes espèces (rat, hamster, chien, lapin et homme). Récemment, nous avons mis en évidence la puissante capacité antilipolytique du peptide YY (PYY) et du neuropeptide Y (NPY) [1].

Ces deux peptides appartiennent, avec le polypeptide pancréatique (PP), à une même famille structurale de peptides régulateurs composés de 36 acides aminés. Leur structure primaire présente un pourcentage d'analogie de $70 \%$ entre le NPY et le PYY, et de $50 \%$ entre le PP et le NPY ou le PYY. Les séquences en acides aminés ont été très conservées au cours de l'évolution. La séquence du NPY est identique chez l'homme, le rat, le cobaye et le lapin et ne diffère de celle du porc que par la présence d'une leucine au lieu d'une méthionine en position 17. Les similarités entre ces trois peptides se retrouvent également dans leurs structures secondaires et tertiaires ainsi que dans l'organisation structurale des précurseurs du NPY, PYY et PP. Les gènes codant pour ces trois peptides ont également été clonés et les similitudes qui lient le NPY, le PYY et le PP laissent supposer l'existence d'un gène ancestral commun à ces peptides (pour revue voir [2]).

\section{Distribution tissulaire et libération du PYY et du NPY}

Le PYY est une hormone intestinale sécrétée par les cellules endocrines de l'épithélium intestinal. Les plus fortes concentrations intracellulaires de PYY se trouvent au niveau de l'iléon et du côlon et même au niveau du rectum chez l'homme [3]. Une importante sécrétion de PYY dans la circulation sanguine a lieu après l'ingestion d'un repas. La concentration de PYY plasmatique augmente alors progressivement à partir de la quinzième minute et reste élevée pendant plusieurs heures. Cependant, l'intensité de la sécrétion de PYY dépend de la nature et de la valeur calorique du repas: plus un repas est énergétique et riche en graisses, plus la sécrétion de PYY est importante [3]. La libération de PYY peut être provoquée par la présence de nutriments ou de sels biliaires au niveau de l'iléon et du colon. Cependant, le délai très bref (15 à 30 minutes) de déclenchement de la sécrétion du PYY se situe bien avant que les nutriments aient pu atteindre l'iléon, ce qui implique également l'intervention d'un signal endocrine et/ou neuronal. Le PYY entraîne des actions inhibitrices dans le tractus gastro-intestinal tant au niveau des sécrétions (d'acide, de pepsinogène, de sécrétine, de CCK...) que de l'absorption et de la motilité intestinales [4].

Le NPY, en tant que neurotransmetteur est localisé aussi bien dans les neurones du système nerveux périphérique que central. Dans le système nerveux autonome, il a été identifié dans l'innervation intrinsè- 
que du pancréas, du tractus gastrointestinal et dans les fibres nerveuses sympathiques périvasculaires [2]. Dans certaines régions du cerveau, le NPY peut être co-localisé avec d'autres neurotransmetteurs tels que la somatostatine et les catécholamines. Le NPY et la noradrénaline (NA) sont co-localisés dans de larges vésicules et sont libérés aux fortes fréquences de stimulation des fibres sympathiques $(>2 \mathrm{~Hz})$. En revanche, la NA contenue dans des vésicules de stockage plus petites est libérée pour de faibles fréquences de stimulation $(<2 \mathrm{~Hz})$.

Le NPY est présent abondamment dans l'hypothalamus et le système limbique, en particulier dans les noyaux arqué et paraventriculaire. Il est impliqué dans de nombreux processus physiologiques et endocrines du métabolisme énergétique. Une des actions centrales les plus puissantes est son effet stimulant sur la prise alimentaire [5].

Plusieurs points pouvaient laisser présager un effet possible de ces peptides sur l'adipocyte dans diverses situations physiologiques:

- Le NPY, ou le PYY, administrés de façon chronique dans le système nerveux central, conduisent à une augmentation des dépôts adipeux et l'obésité en est la principale conséquence [6].

- Le NPY et la noradrénaline coexistent à l'intérieur d'une même terminaison nerveuse dans le système sympathique périphérique et ces neurotransmetteurs peuvent être co-libérés dans certaines conditions de stimulation.

- Des interactions entre les effets $\alpha_{2}$-adrénergiques et ceux du NPY ont été décrites dans le système sympathique [7].

- L'exercice physique prolongé entraîne une augmentation de l'activité sympathique qui active la lipolyse. Lundberg et al. ont montré que le NPY est co-libéré avec les catécholamines lors de l'exercice physique [8].

- Dans le tissu adipeux brun, les fibres nerveuses orthosympathiques innervant les vaisseaux contiennent aussi du NPY. En effet, la sympathectomie chi- mique induite par la 6-hydroxydopamine entraîne à la fois la déplétion en noradrénaline, classiquement décrite, mais aussi la disparition de l'immunoréactivité au NPY [9].

Notons également que l'une des voies de transduction du signal pour les récepteurs du PYY et du NPY est l'inhibition de l'activité de l'adénylyl cyclase, enzyme clé de la régulation de la lipolyse. Connaissant l'implication du système adrénergique dans le métabolisme du tissu adipeux, l'existence d'une interaction entre ces peptides et la cellule adipeuse a été recherchée.

\section{Le PYY et le NPY: inhibiteurs de la lipolyse}

Le NPY et le PYY inhibent la lipolyse de manière dépendante de la dose dans des adipocytes isolés canins et humains. Lorsque les cellules sont prétraitées à la toxine pertussique qui inactive, par ADP-ribosylation, la sous-unité $\alpha i$ des protéines $\mathrm{Gi}$, les effets inhibiteurs de ces peptides sont abolis [1].

Les effets antilipolytiques du NPY et du PYY ont également été recherchés dans différentes espèces. Sur des adipocytes de lérot, un rongeur hibernant, ces deux peptides inhibent la lipolyse de façon maximale [10]. En revanche, il s'est avéré que, dans nos conditions d'étude, chez les animaux de laboratoire couramment utilisés (rat, hamster, cobaye et lapin), les réponses antilipolytiques du NPY et du PYY sont faibles ou inexistantes. Au vu des

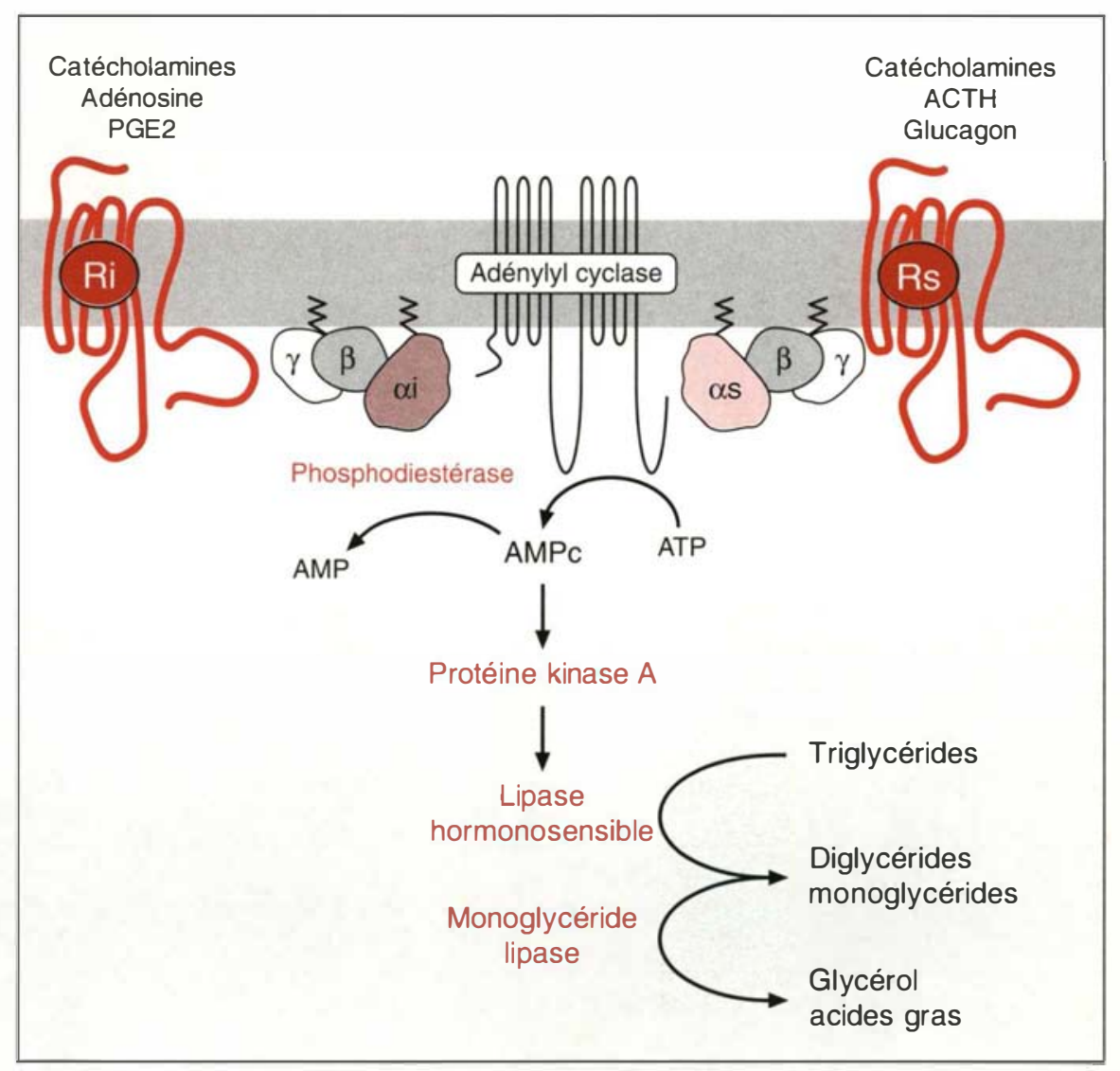

Figure 1. Représentation des divers éléments du système adénylcyclasique de la membrane plasmique adipocytaire: les récepteurs stimulant (Rs) ou inhibant (Ri) l'adénylyl cyclase; les protéines G (Gs et Gi) composées des sous-unités $\alpha$ ( $\alpha$ j ou $\alpha$ s), $\beta$ et $\gamma$; l'adénylyl cyclase, protéine transmembranaire contrôlant la production d'AMP cyclique. 


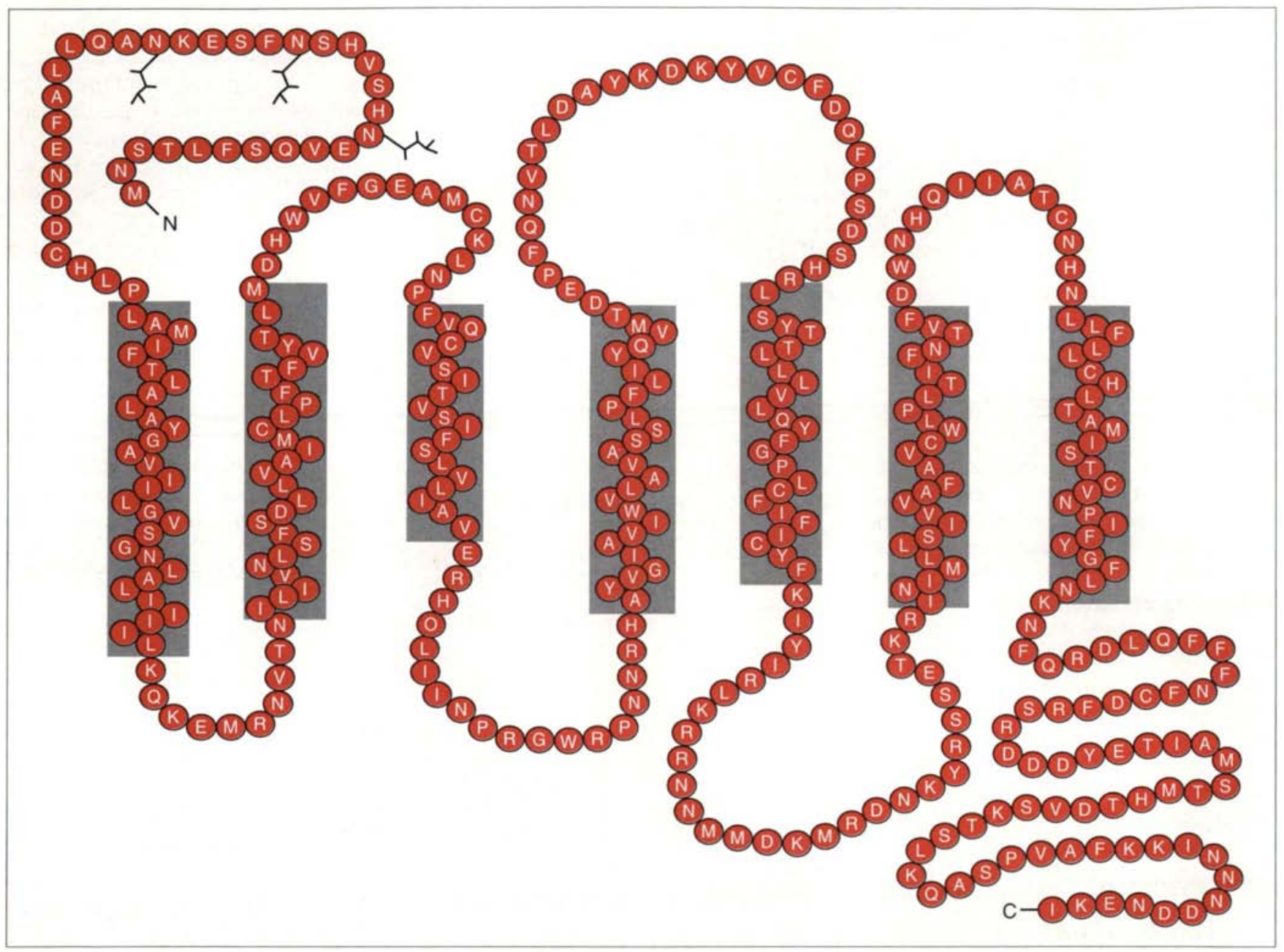

Figure 2. Représentation topographique du récepteur du NPY, d'après Wahlestedt, et al. [19]. Les passages transmembranaires sont représentés par les rectangles gris.

Tableau I

CARACTÉRISTIQUES BIOCHIMIQUES DES DIVERS SOUS-TYPES DE RÉCEPTEURS DU NPY ET DU PYY

\begin{tabular}{|c|c|c|c|c|}
\hline Sous-type & Agoniste & Localisation & $\begin{array}{l}\text { Poids } \\
\text { moléculaire }\end{array}$ & $\begin{array}{l}\text { Second } \\
\text { messager }\end{array}$ \\
\hline Y1 & $\begin{array}{l}\left.\text { [Leu }{ }^{31}-\mathrm{Pro}^{34}\right] \mathrm{NPY} \\
\text { NPY } \\
\text { PYY }\end{array}$ & $\begin{array}{l}\text { Neuroblastome } \\
\text { Cerveau }\end{array}$ & $\begin{array}{l}70 \mathrm{kDa} \\
62 \mathrm{kDa}\end{array}$ & $\begin{array}{l}\mathrm{Ca}^{2+} \\
\mathrm{AMPc}\end{array}$ \\
\hline Y2 & $\begin{array}{l}(13-36) \text { NPY } \\
\text { NPY } \\
\text { PYY }\end{array}$ & $\begin{array}{l}\text { Intestin } \\
\text { Rein } \\
\text { Cerveau } \\
\text { Tissu adipeux }\end{array}$ & $\begin{array}{l}44 \mathrm{kDa} \\
50 \mathrm{kDa} \\
62 \mathrm{kDa} \\
62 \mathrm{kDa}\end{array}$ & AMPc \\
\hline Y3 & $\begin{array}{l}\left.\text { [Pro }{ }^{34}\right] \text { NPY } \\
\text { NPY }\end{array}$ & $\begin{array}{l}\text { Cellules } \\
\text { chromaffines } \\
\text { (lignée) }\end{array}$ & ND & $\mathrm{Ca}^{2+}$ \\
\hline
\end{tabular}


résultats obtenus chez le chien et l'homme, nous avons alors identifié et caractérisé les récepteurs mis en jeu.

\section{Les récepteurs du PYY et du NPY}

Ce sont des récepteurs métabotropiques (protéines à sept passages transmembranaires, couplés aux protéines $\mathrm{G}$ ), présentant des sites de $\mathrm{N}$ glycosylation. Il existe une distinction entre les récepteurs du NPY et du PYY. Ces derniers présentent une meilleure affinité pour le PYY que pour le NPY. L'utilisation de fragments du NPY et du PYY ou d'analogues de synthèse a permis de connaître les parties du peptide impliquées dans l'activation du récepteur, mais aussi de distinguer les divers sous-types de récepteurs du NPY et du PYY. Ainsi, les récepteurs sont considérés comme étant du soustype Y2 lorsque le fragment Cterminal, le NPY(13-36), entraîne une meilleure réponse que les analogues de synthèse du NPY tels que le $\left[\mathrm{Leu}^{31}-\mathrm{Pro}^{34}\right] \mathrm{NPY}$ ou le $\left[\mathrm{PrO}^{34}\right]$ NPY. Les récepteurs du sous-type YI ont, en revanche, une meilleure affinité pour les analogues de synthèse que pour le fragment C-terminal. Des récepteurs appartenant au soustype Y3 ont également été décrits, ils ont la particularité de ne présenter aucune affinité pour le PYY[11]. Pour l'instant, seul le gène codant pour le sous-type Yl a été cloné chez l'homme [12]. La séquence en acides aminés est représentée sur la figure 2.

\section{Les récepteurs du PYY du tissu adipeux}

La caractérisation de ces récepteurs a d'abord été effectuée chez le chien puis chez l'homme, modèles où les effets antilipolytiques les plus puissants du NPY et du PYY avaient été obtenus. Dans la cellule adipeuse de chien, nous avons pu mettre en évidence l'implication d'un récepteur du PYY post-synaptique, de type Y2 et d'un poids moléculaire apparent de $62 \mathrm{kDa}$, dans les processus antilipolytiques induits par le PYY et le NPY. Ces récepteurs sont $\mathrm{m} / \mathrm{s} n^{\circ} 2 \mathrm{vol}$. 10, féuriet 94 couplés à des protéines Gi car, d'une part, ils inhibent l'activité de l'adénylyl cyclase et, d'autre part, la toxine pertussique supprime l'effet antilipolytique [13]. Des récepteurs du PYY (sous-type Y2) ont également été décrits dans plusieurs préparations membranaires telles que les cellules du tube proximal du rein (lapin) [14], les cellules de l'épithélium intestinal (rat) [15] ou encore les cellules principales de l'estomac (cobaye) [16] (Tableau I).

\section{Le tissu adipeux humain}

Le tissu adipeux humain est réparti différemment selon le sexe. Ainsi, chez la femme, il a tendance à se développer dans la région fémorale et, chez l'homme, dans la région abdominale. Cette caractéristique morphologique permet de définir respectivement les profils gynoïdes et androïdes. De plus, selon sa localisation anatomique [profond (omentum) et sous-cutané (fémoral,

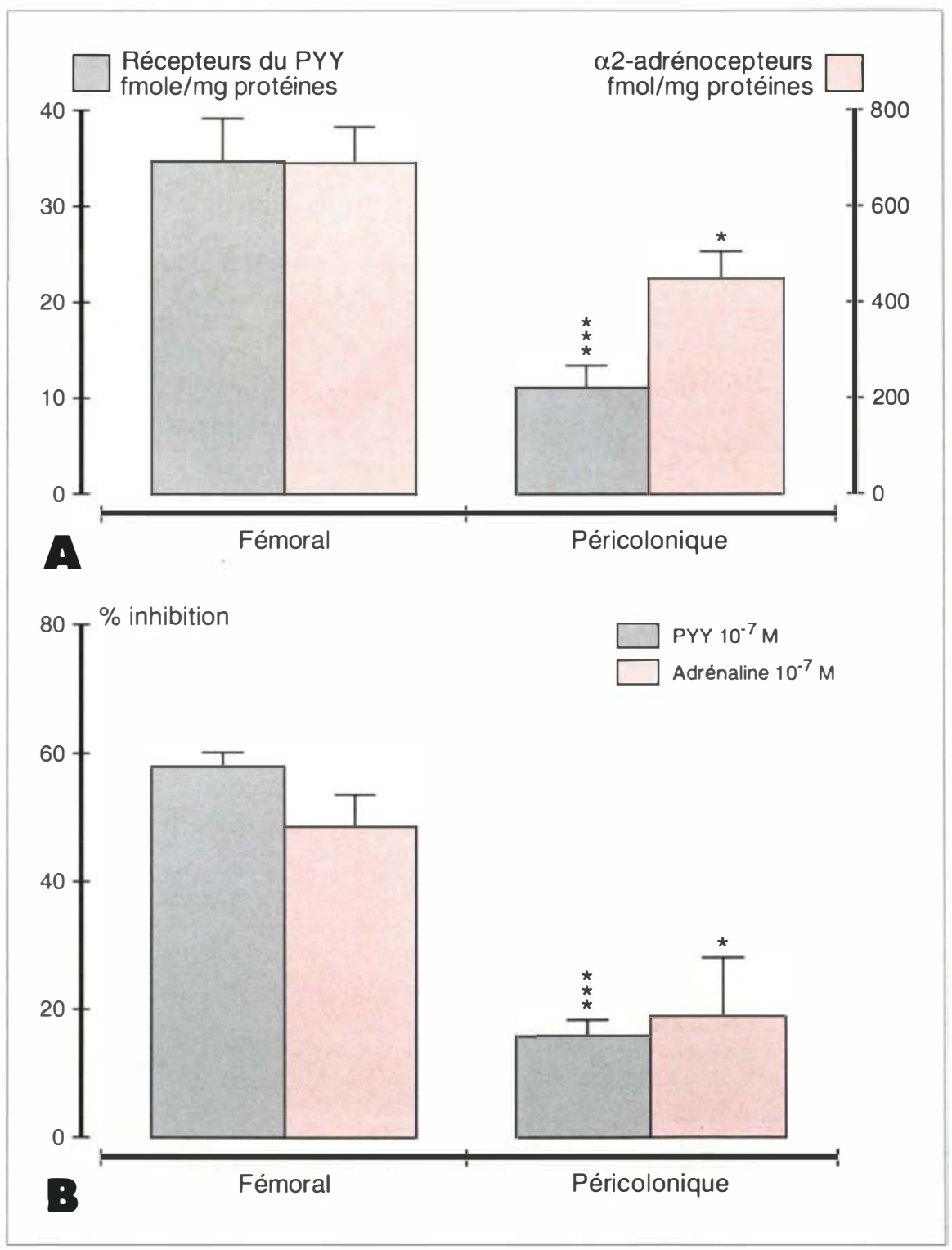

Figure 3. Étude comparée de la réceptivité $\alpha_{2}$-adrénergique et peptidergique dans le tissu adipeux fémoral et péricolonique. A : expression des récepteurs. B: inhibition de la lipolyse par le PY et l'adrénaline. $x: p<0,05 ; x x x: p<0,001$ 
abdominal)], le tissu adipeux n'a pas les mêmes facultés lipomobilisatrices. En effet, l'étude des effets de l'adrénaline sur des adipocytes isolés de trois dépôts adipeux (fémoral, abdominal et omental) a montré que cette hormone lipolytique dans l'omentum, devient strictement antilipolytique dans le tissu fémoral et exerce un effet biphasique dans le tissu abdominal [17]. La différence de réponse à l'adrénaline est fonction du niveau d'expression des récepteurs $\beta$-adrénergiques (lipolytiques) et $\alpha_{2}$-adrénergiques (antilipolytiques). Un équilibre fonctionnel $\alpha_{2} / \beta$ adrénergique a été bien mis en évidence. L'adrénaline exerce des effets antilipolytiques dans les dépôts sous-cutanés oú le nombre de récepteurs $\alpha_{2}$-adrénergiques est nettement supérieur à celui des récepteurs $\beta$-adrénergiques. Dans les tissus profonds, où le rapport $\alpha_{2} / \beta$ est inférieur à un, l'adrénaline est lipolytique. Remarquons

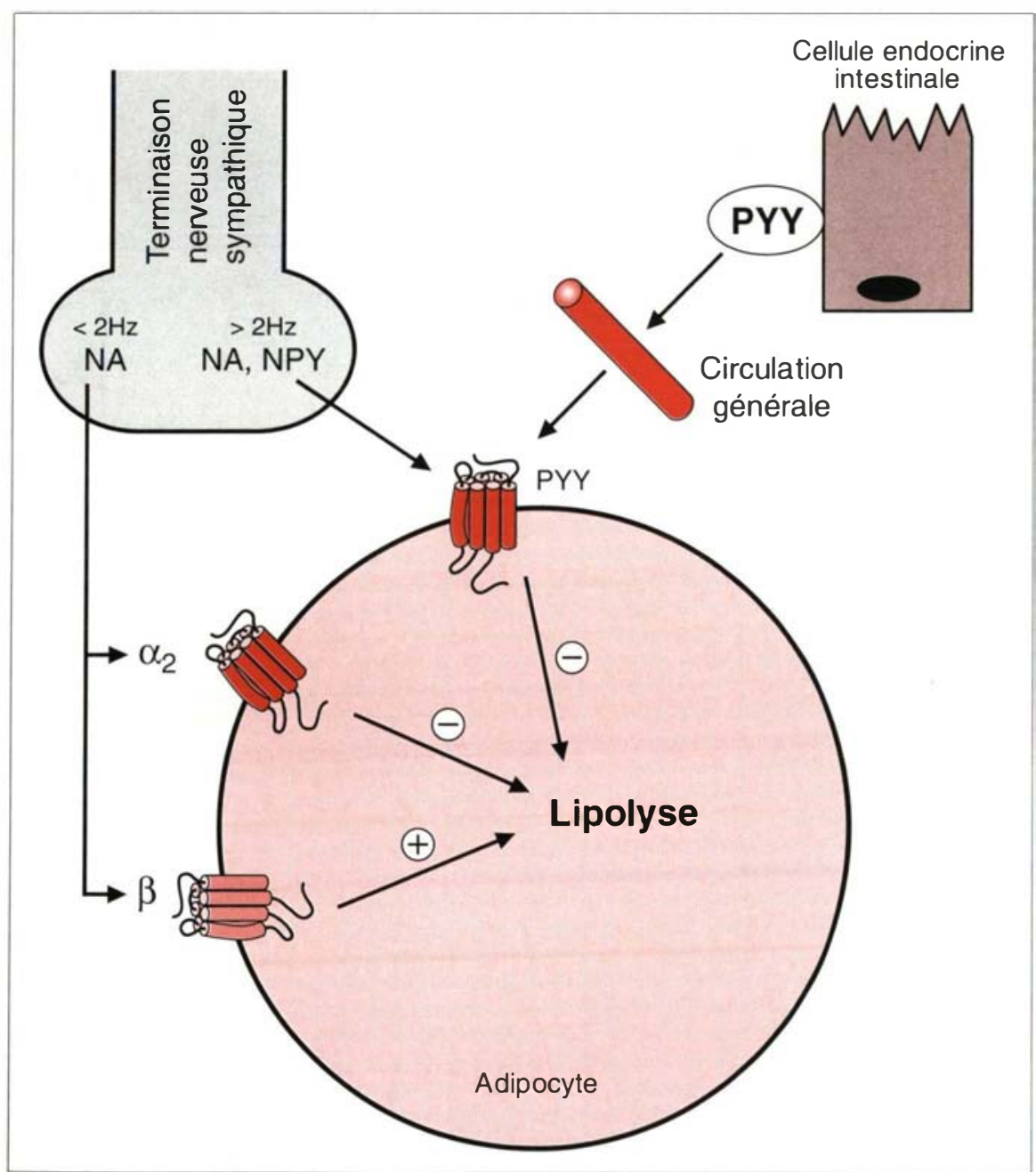

Figure 4. Représentation schématique des axes de régulation neuroadipocytaire et entéro-adipocytaire. NA: noradrénaline; Hz: Hertz. que ces effets dépendent grandement de la concentration de catécholamines. En effet, les récepteurs $\alpha_{2}$ et $\beta$-adrénergiques sont recrutés de façon différentielle selon leurs affinités respectives pour l'agoniste physiologique.

Il était donc intéressant de rechercher si le système PYY/NPY (nombre de récepteurs et capacité d'inhibition de l'adénylyl cyclase) présentait aussi une efficacité variable en fonction de la localisation du tissu adipeux. Comme pour le récepteur $\alpha_{2}$-adrénergique, le nombre de récepteurs du PYY est beaucoup plus faible dans les tissus profonds que dans les tissus sous-cutanés (figure 3A). Une étroite corrélation existe même entre le niveau d'expression des récepteurs $\alpha_{2}$-adrénergiques et du PYY dans le tissu adipeux fémoral. Par voie de conséquence, l'effet antilipolytique du PYY et du NPY varie également en fonction du dépôt adipeux étudié: l'inhibition maximale de la lipolyse a lieu dans les dépôts souscutanés alors que seul un effet résiduel persiste dans les tissus profonds (figure 3B) [18]. Ces récepteurs, $\alpha_{2}$-adrénergiques et du PYY, par leur abondance dans les adipocytes des tissus adipeux sous-cutanés, semblent avoir un rôle déterminant dans le maintien d'une mobilisation lipidique réduite.

\section{Perspectives}

La caractérisation des récepteurs du PYY dans le tissu adipeux (humain et canin) a permis de confirmer l'existence d'un nouveau système antilipolytique s'ajoutant à trois autres puissants systèmes antilipolytiques caractérisés antérieurement (récepteurs $\alpha_{2}$-adrénergiques, $\mathrm{Al}$ de l'adénosine è de la prostaglandine E2). Pour l'instant, on ne connaît pas encore toutes les caractéristiques fonctionnelles des récepteurs du PYY. Il reste en effet à confirmer, sur un plan génique, l'existence des différents sous-types de récepteurs du NPY ou du PYY; d'ailleurs, le gène codant pour le récepteur Y2 n'a pas encore été cloné. Les futurs travaux sont suceptibles de révéler, 
comme pour d'autres familles d'hormones et de neurotransmetteurs, une hétérogénéité plus importante des récepteurs du NPY et du PYY. En ce qui concerne les outils pharmacologiques et les médicaments potentiels, de véritables antagonistes des récepteurs du NPY et du PYY font encore défaut. La recherche de composés antagonistes non peptidiques constitue un développement essentiel pour l'exploration et la compréhension des mécanismes.

Les études que nous avons réalisées sur des adipocytes isolés, ne permettent pas encore d'intégrer les effets du NPY et du PYY dans la physiologie de la mobilisation lipidique. Des travaux sont également nécessaires pour comprendre la contribution relative des divers systèmes antilipolytiques de l'adipocyte humain ainsi que les situations physiologiques propices à la mise en jeu du système PYY/NPY. Pour conceptualiser cette question, il faut souligner que le NPY et la noradrénaline sont co-localisés dans certaines terminaisons nerveuses sympathiques et colibérés pour de fortes fréquences de stimulation, ce qui suggère l'implication d'un axe de régulation neuro-adipocytaire. De plus, le fait que le PYY, hormone intestinale sécrétée de façon soutenue à la suite d'un repas, agisse sur la cellule adipeuse, va permettre d'envisager et d'étudier un axe de régulation entéro-adipocytaire tout à fait original et totalement inexploré (figure 4)
1. Valet P, Berlan M, Beauville M, Crampes $F$, Montastruc J, Lafontan M. Neuropeptide Y and peptide YY inhibit lipolysis in human and dog fat cells through a pertussis toxin sensitive G-protein. J Clin Invest $1990 ; 85: 291-5$.

2. Dumont Y, Martel JC, Fournier A, St Pierre S, Quiron R. Neuropeptide Y and neuropeptide $Y$ receptor subtypes in brain and peripheral tissues. Prog Neurriol 1992 ; 38: $125-67$

$\mathrm{m} / \mathrm{s} n^{\circ} 2 \mathrm{vol}$. 10 , férrier 94
3. Adrian T, Ferri G, Bacarese-Hamilton A, Fuessl H, Polak J, Bloom S. Human distribution and release of a putative new gut hormone, peptide YY. Gastroenterology' 1985; 89: 107()-7.

4. Taylor I. Peptide YY: the ileo-colonic, gastric, and pancreatic inhibitor. Biol Bull $1989 ; 177$ : 187-91.

5. Leibowitz S. Brain neuropeptide Y: an integrator of endocrine, metabolic and behavioral processes. Brain Res Bull 1991 ; 27 : 333-7.

6. Stanley B, Kyrkouli S, Lampert S, Leibowitz S. Neuropeptide Y chronically injected into the hypothalamus: A powerful neurochemical inducer of hyperphagia and obesity. Peptides 1986; 7: 1189-92.

7. Dahlöf C, Dahlöf P, Lundberg J. $\alpha_{1}$-adrenoceptor-mediated inhibition of nerve stimulation-evoked release of neuropeptide Y (NPY)-like immunoreactivity in the pithed guinea-pig. Eur I Pharmacol 1986 ; 131 : 279-83.

8. Lundberg J, Martinsson A, Hemsen A, Theodorsson-Norheim E, Svedenhag J, Ekblom B, Hjemdahl P. Co-release of neuropeptide $\mathrm{Y}$ and catecholamines during physical exercise in man. Biochem Biophys Res Commun 1985; 133: 30-6.

9. Cannon B, Nedergaard J, Lundberg J, Hokfelt T, Terenius L, Goldstein M. Neuropeptide tyrosine (NPY) is costored with noradrenaline in vascular but not in parenchymal sympathetic nerves of brown adipose tissue. Exp Cell Res 1986; 164: 546-50.

10. Castan I, Valet P, Quideau N, Voisin T, Ambid L, Laburthe M, Lafontan M, Carpéné C. Antilipolytic effects of alpha2-agonists, neuropeptide $\mathrm{Y}$, adenosine and PGE 1 in various mammal species. $A m$ J Physiol (sous presse).

11. Wahlestedt C, Regunathan S, Reis D. Identification of cultured cells selectively expressing Y1-, Y2-, or Y3-type receptors for neuropeptide Y/peptide YY. Iife Sci 1992 50: $7-12$.

12. Sheikh S, Hansen A, Williams J. Solubilization and affinity purification of the Y2 receptor for neuropeptide $\mathrm{Y}$ and peptide YY from rabbit kidney. I Biol Chem 1991; 266 : 23959-66.

15. Herzog H, Hort Y, Bali H, Hayes G, Shine J, Selbie L. Cloned human neuropeptide Y receptor couples to two different second messager system. Proc Natl Acad Sa USA 1992; 89: 5794-8.

16. Castan I, Valet P, Voisin T, Quideau N, Laburthe M. Lafontan M. Identification and functional studies of a specific peptide YY-preferring receptor in dog adipocytes. Endocrinology 1992; 131 : 1970)-6.

17. Mauriège $P$, Galitzky J, Berlan $M$ Lafontan M. Heterogenous distribution of beta- and alpha2-adrenoceptors binding sites in human fat cells from various deposits. Functional consequences. Eur J Clin Invest $1987 ; 17: 156-65$.

18. Castan 1, Valet P, Larrouy D, Voisin T, Remaury A, Daviaud D, Laburthe $M$, Lafontan M. Distribution of PYY receptors in human fat cells : an antilipolytic system alongside the alpha2-adrenergic one. $\mathrm{Am}$ J Physiol 1993; 265: 74-80.

19. Wahlestedt C, Reis DJ. Neuropeptide Y-related peptides and their receptors : are the receptors potential therapeutic drug targets? Annu Rey Toxicol 1993; 33: 309-52.

\section{Isabelle Castan}

Attachée temporaire d'enseignement et de recherche.

\section{Philippe Valet}

Maître de conférence université Paul-Sabatier

\section{Max Lafontan}

Directeur de recherche à l'Inserm Régulation adrénergique et adaptations métaboliques, Inserm U317, Institut Louis-Bugnard, CHU Rangueil, bâtiment L,3, 31054 Toulouse Cedex, France.

13. Voisin T, Couvineau A, RouyerFessard C, Laburthe M. Solubilization and hydrodynamic properties of active peptide YY receptor from rat jejunal crypts. Characterization as a $\mathrm{Mr} 44,000$ glycoprotein. J Biol Chem 1991; 266: 10762-7.

14. Singh G, Singh L, Raufman J. Y2 receptors for peptide $\mathrm{YY}$ and neuropeptide $\mathrm{Y}$ on dispersed chief cells from guinea pig stomach. Am / Physiol 1992; 25: 756-62.

\section{TIRÉS A PART}

I. Castan. 